Prísclla Bernardina MiRanda SOARES

ORIENTADOR:

\title{
Avaliação clínico-epidemiológica e qualidade de vida em mulheres com câncer de mama
}

Prof. Dr. Hercílo Martelli JúNior

\author{
Clinical and epidemiological evaluation and quality of life in women with \\ breast cancer
}

\section{Resumo de tese}

Palavras-chaves

Câncer de mama Diagnóstico tardio Qualidade de vida

Keywords

Breast cancer Late diagnosis Quality of life
Dissertação de Mestrado apresentada ao Programa de Pós-Graduação em Ciências da Saúde da Universidade Estadual de Montes Claros, Minas Gerais, para obtenção do Título de Mestre, em 08 de dezembro de 2010.

OBJETIVOS:avaliar perfil clínico-epidemiológico de Serviços de referência oncológica - um público e um privado e comparar qualidade de vida entre mulheres já tratadas do câncer de mama e mulheres sem câncer. MÉTODOS: realizouse estudo transversal avaliando 288 prontuários de pacientes com câncer de mama admitidas entre 2006 e 2009. Variáveis foram analisadas pelos testes qui-quadrado, regressão logística binária e multinomial. Feito também estudo caso-controle, utilizando o SF-36 para avaliar qualidade de vida entre mulheres com e sem câncer de mama entre 40 e 69 anos, sendo 70 com a doença e 84 sem câncer. Análises foram feitas pelo teste qui-quadrado e modelo linear multivariado. RESULTADOS: ao comparar os Serviços público e privado, 53,6\% das admitidas no serviço público apresentavam diagnóstico tardio (estágios III e IV), assim como o tempo entre suspeita e confirmação diagnóstica acima de 6 meses (54, 1 versus 18,5\%). Já a realização de mamografia foi mais frequente no privado (54,3 versus 34,7\%). Scores médios dos componentes físico e mental do SF-36 foram discretamente superiores nas sobreviventes ao câncer de mama (51, 10 e 52,25 respectivamente) comparados ao controle (47,26 e 47,93 respectivamente). CONCLUSÃO: houve maior prevalência de pacientes em estágios mais avançados, maior tempo entre suspeita e confirmação diagnóstica e menor frequência de realização de mamografia no Serviço público quando comparado com o privado. Sobreviventes ao câncer de mama tiveram melhores indicadores de qualidade de vida no componente físico e no mental. 